\title{
Effectiveness of a Portable Dental Hygiene Outreach Program in Improving the Oral Health of Disabled Adults
}

\author{
Roberto Ferro ${ }^{1}$, Valerio Dainese ${ }^{2}$, Luca Benacchio ${ }^{3}$, Alberto Besostri ${ }^{4}$, Armando Olivieri ${ }^{5}$
}

\begin{abstract} attending daytime centers for disabled (DCD). A trend in $\mathrm{Bl}$ across groups was evaluated. significant) improvement in each level of the chosen index was recorded. sessions.

"It is estimated that one out of two persons with a significant disability cannot find a professional resource to provide appropriate and necessary dental care. Lack of access to dental services for this growing segment of our population is reaching critical levels and is a national dilemma."
\end{abstract}

Aim and objective: The aim of the article was to assess the effectiveness of a portable outreach oral hygiene program for people with disabilities

Materials and methods: Professional oral hygiene sessions were provided at the DCDs using a portable dental equipment in the Local Health Unit (LHU) n.6-Veneto Region-Italy. Patients were grouped by the total number of sessions performed. Clinical primary outcome was bleeding on probing (BoP) index. We compared mean bleeding index (BI) among subjects groups at baseline and at the end of the follow-up (2009-2016).

Results: From May 2009 to March 2016, 345 subjects were routinely examined and treated regarding their oral hygiene needs. A clear (statistically

Conclusions: The program is effective in improving the oral health in this underserved population.

Clinical significance: This portable outreach program provides a measurable progressive improvement in the periodontal health; a progressive time reduction of each session; a facilitated access to oral health treatment; and an augmented compliance of the oral hygiene

Keywords: Cohort study, Special care dentistry, Access to dental care, Patient outcomes, Dental hygiene, Bleeding on probing.

Journal of Oral Health and Community Dentistry (2020): 10.5005/jp-journals-10062-0079

\section{INTRODUCTION}

Adults with disabilities comprise a heterogeneous population manifesting a wide array of disabling conditions and degrees of severity of impairment. Many special care patients (SCPs) have severe dental decay or periodontal disease related to their medical condition or developmental disabilities (e.g., high consumption of medications and cariogenic diet). ${ }^{2-4}$ The major differences for the group with disability were a higher number of untreated carious lesions, lack of oral care, and infrequent use of preventive strategies. ${ }^{5,6}$ In the USA, dental care is the second-most frequent unmet need in individuals with cognitive deficiencies, physical limitations, and/or behavior problems that compromise self-care and may require additional management. ${ }^{7}$ Periodontal disease is one of the important prevalent oral diseases among the disabled-adult population, the main cause of periodontal disease being the inability to administer personal oral hygiene care with different degrees based on the kinds of disabilities. ${ }^{1}$ Chung et al. ${ }^{8}$ reported that disabled persons showed a poorer oral hygiene status as compared to the nondisabled and the general need for plaque control and oral prophylaxis. Thus, professional help for oral hygiene care is required for all kinds of disabled persons, according to the kinds of handicap. Shin et al. ${ }^{9}$ reported that factors such as the age, type of disability, and tooth-brushing ability, could influence the periodontal health status of disabled persons. In a recent study of adolescents and adults, the authors

\begin{abstract}
${ }^{1,2,4}$ Dental Unit, Health District n.6, Cittadella, Padua, Italy
${ }^{3}$ Department of Prevention, Health District n.6, Padova, Padua, Italy

${ }^{5}$ Epidemiologist, Former Department of Prevention, Health District n.6, Padua, Italy
\end{abstract}

Corresponding Author: Alberto Besostri, Department of Dental Unit, Health District n.6Veneto Region, Cittadella, Italy, Phone +393389225464, e-mail: albertobesostri1@gmail.com

How to cite this article: Ferro R, Dainese V, Benacchio L, et al. Effectiveness of a Portable Dental Hygiene Outreach Program in Improving the Oral Health of Disabled Adults. J Oral Health Comm Dent 2020;14(3):88-92.

Source of support: Nil

Conflict of interest: None

observed that patients with intellectual disability had more decayed and missing teeth, fewer restorations, and a greater need for tooth extraction. ${ }^{10}$

Access to affordable and acceptable health care is a basic human right, ${ }^{11}$ but, unfortunately, an inverse relationship exists in this context, and people who have greater health needs are the ones who receive the least amount of care. ${ }^{12}$ Many barriers exist to access oral health care in the population with disabilities. The limitations in access to dental care are well documented and include such factors as financial disincentives, limited availability of trained dental personnel, and location of treatment facilities resulting in a high burden of dental diseases. ${ }^{10,13}$

In Italy, people with disabilities are 13,177,000 and about 3 million of these subjects need more intensive support. ${ }^{14}$ In Italy, the access to dental care has declined throughout the economic crisis years and the Italian National Health System grants the $5 \%$ of 
the dental care delivery, being most of the dental services out of pocket. All indicators of access to dental care and oral health show inequalities related to socioeconomic status. ${ }^{15}$

The recent report of the state of health and the quality of the healthcare delivery in the 21 Italian regions ${ }^{16}$ points out that the indicator of the unperformed health needs in people with disability - 3,167,000 persons in 2013-is related to financial difficulties, particularly regarding oral health treatments. ${ }^{17}$ This indicator supplies a regional survey of the people declaring to have renounced to dental care with financial difficulties but in the presence of daily activity limitations compared to those without limitations. A very marked difference was discovered between these two segments of the population: 22 vs $8.3 \%$ (Italy) and 10.9 vs $4.2 \%$ (Veneto region). ${ }^{16}$

To face these oral health inequalities, in 2009, we planned a portable outreach oral health program for people with disabilities attending daytime centers for disabled (DCD) in the Health District $n^{\circ} 6$ in the Veneto Region of Italy adopting the criteria defined by the Association of State and Territorial Dental Directors: ${ }^{18}$

- Impact/Effectiveness: A practice or program produces outcomes that improve the oral health status and/or improve access to dental care for with SCPs. A practice or program enhances the processes to improve the oral health status and/or improve access to dental care for SCPs;

- Efficiency: A practice or program shows cost savings in preventing oral disease and reducing the extent of treatment needs for SCPs;

- Demonstrated sustainability; and

- Collaboration/Integration: The program offers regular/routine appointments and appropriate treatment provided directly in the centers by a dental hygienist.

The aim of the study was to assess the effectiveness of such an oral hygiene program in terms of oral health as a primary outcome and as secondary outcomes, efficiency, sustainability, collaboration/integration, and compliance by SCPs, caregivers, tutors, and parents.

\section{Study Population and Methodology}

A prospective observational cohort study was conducted in 12 DCDs spreading in the area of Local Health Unit (LHU) n.6, after its design was granted approval by the Local Ethics Committee.

Authorization was obtained by the administrations of the DCDs.

A written consent form was signed from the parents of the people attending DCDs with agreement to the treatment plan in line with the guidelines of the Declaration of Helsinki.

People attending DCDs were mainly affected by mental disability (36.5\%), Down's syndrome (14\%), seizure disorders (8\%), and tetraparesis (6\%). Many people (35.5\%) accounted for more than one health problem.

We recruited all subjects attending DCDs on the examination day for a first visit/control assessing their oral hygiene status (baseline).

Patients were visited after baseline to plan a different number of visits/oral health sessions according to their oral hygiene status. For each subject, the data were entered into an electronic case report. All codes and definitions were established before starting the program and the following data were collected for each SCP at baseline: demographic data, selected systemic diseases (e.g., diabetes), pharmacological treatments (antiepileptic drugs), and lifestyles (smoking) that could harm oral health. The primary outcome was bleeding on probing (BoP) index ${ }^{19}$ at the gingival margin at baseline and at every control visit after baseline. Bleeding on probing was assessed by means of bleeding index $(\mathrm{BI})$, based on recording bleeding on each of the four surfaces of the teeth (lingual, buccal, mesial, and distal) sliding the probe around the tooth. The index is a percentage value defined as the ratio ( $n^{\circ}$ of bleeding surfaces ) $/\left(n^{\circ}\right.$ of total surfaces) $\times 100$. The follow-up took part from January 5, 2009, to March 31, 2016 (respectively, first and last visit available). We censored data accounting for people with a baseline visit date no longer than September 30, 2015 (6 months to last visit available), to allow the detection of a clinical improvement. The program was designed to provide routine visits/oral health sessions at the DCDs keeping up-to-date a registry about people attending DCDs and their oral hygiene status. Therefore, people could have more than one visit recorded regardless of their visit outcome. We identified the cohort for this study keeping in mind the aim and the primary outcome:

- For people with more than one visit, each one detecting a value of $\mathrm{BI}=0$, only the first record (first visit) was included;

- When the value of $\mathrm{BI}=0$ was reached in a sequence of visits, we considered that last one as the last visit (regardless of the registry kept on recording more controls); otherwise, $\mathrm{BI}$ value in the last visit was included.

Furthermore, six subjects were excluded because the lag time from baseline and first or second control lasted more than 36 months (for people with no more than 3 visits).

\section{Oral Hygiene Program}

Professional oral hygiene sessions are provided at DCDs using a mobile dental equipment (portable dental unit, portable chair, portable dental LED light, and portable tray) and sterile and/or disposable dental instruments. All the SCPs, after being sat on the portable or their own chair, are visited, including the edentulous ones, to assess the oral health status. All the clinical parameters are assessed by one trained experienced examiner under standard dental office and light source conditions. In each step of the survey, all the clinical parameters are assessed by the only one trained experienced examiner. Prior to the beginning of this outreach program, to assess the intra-examiner reliability, 30 visits on SCPs attending our dental unit were performed and then repeated after one month with a resulting $K$ value of $85 \%$. Subjects are clinically examined and treated at the control visit(s) in the same way as at baseline. At each oral hygiene appointment, the dental hygienist examines their gingival status. He gives the necessary course of treatment and advice to help to maintain a good gingival status and to prevent re-occurrence. During the hygiene session, the dental hygienist removes plaque, tartar, and stains. Once all the deposits have been removed, the dental hygienist places on an ongoing preventative maintenance program of three, four, or six monthly recall intervals, depending on the individual needs. During the course of treatment, the dental hygienist gives dental health education. This involves showing and advising the daily oral hygiene practices to keep the plaque levels to a minimum not only to SCPs, but also to their parents and, before the beginning of this 
project, dental health education meetings took place involving the residential care staff. After all, as a result of this experience, an oral hygiene instruction booklet was written and then handed out in each DCD.

\section{Statistical Analysis}

The primary outcome of the study was the BoP as recorded during each visit. Descriptive statistics were computed for all the variables: Continuous data are presented as mean \pm standard deviation, and categorical data are presented as counts (percentages). Confidence intervals were estimated at 95\% (95\% $\mathrm{Cl})$. Subjects were grouped by the total number of visits performed at the end of the study period. We estimated mean $\mathrm{BI}$ at baseline and at the end of follow-up, and mean reduction of the same index from baseline to the end of the follow-up period. We assessed/compared mean $\mathrm{BI}$ among subjects groups at baseline and at the end of the follow-up. As BI showed a non-normal distribution, the statistical significance of differences within groups, it was determined by means of nonparametric analysis using Wilcoxon matched-pairs signed-rank test. To estimate a trend in $\mathrm{BI}$ occurrence across groups (between baseline values and mean reduction from baseline to the end of the follow-up), we performed a nonparametric test for trend. A $p$-value $<0.05$ was considered significant. The data were analyzed using Stata rel.14.2 (Stata Corporation, College Station, TX, USA).

\section{Results}

\section{The Study Cohort}

From May 2009 to March 2016, 345 subjects (53\% male) were visited and treated. In Table 1, the characteristics of the cohort are described. Four subjects had diabetes and only three were smokers.

A total of 107 people (31.0\%) assumed antiepileptic drugs, a class of compounds that could induce gingival hyperplasia.

\section{Grouping Patients by Visits}

We performed 922 visits: Patients were divided into six groups according to the number of visits (Table 2). At the beginning of the program, some patients showed low BoP values (146 subjects, 42.3\%) and received only one visit with treatment and advice.

Growing up the periodontal lesions (as indicated by BoP), 58\% of the subjects required two or more and $26 \%$ more than three oral hygiene sessions to improve their status.

\section{Clinical Improving: Bleeding on Probing Course}

Mean BoP at baseline increased from 1-visit group (6.7) to the 6-and-more visits one (54.0): We found a significant trend across groups ( $p<0.001$ ) (Table 3). Whichever the level of the index at the beginning of the program, a clear improvement has been seen for every group (Table 3 ). Accounting for subjects who received more than one intervention, overall mean BoP decreased from 43.2 to 2.9 , and the difference was highly statistically significant ( $p<0.001$ ). Within each group, we could observe a comparable trend: Mean reduction from baseline to last visit was highly statistically significant too. Groups with the largest number of visits/sessions achieved an improvement more pronounced than people with less visits (Table 3 ).

Mean BoP differences (from baseline to last visit) showed a positive trend $(p<0.001)$ across groups.
Table 1: Characteristics of the study population

\begin{tabular}{|c|c|c|}
\hline Characteristics & Freq. $(n=345)$ & $\%$ \\
\hline \multicolumn{3}{|l|}{ Gender } \\
\hline Male & 183 & 53.0 \\
\hline Female & 156 & 45.2 \\
\hline Not recorded & 6 & 1.7 \\
\hline \multicolumn{3}{|l|}{ Age group } \\
\hline-24 & 71 & 20.6 \\
\hline $25-34$ & 73 & 21.2 \\
\hline $35-44$ & 104 & 30.1 \\
\hline $45-54$ & 67 & 19.4 \\
\hline $55-64$ & 22 & 6.4 \\
\hline Not recorded & 8 & 2.3 \\
\hline \multicolumn{3}{|l|}{ Missing teeth } \\
\hline 0 & 28 & 8.1 \\
\hline $1-5$ & 145 & 42.0 \\
\hline $6-15$ & 125 & 36.2 \\
\hline $16+$ & 47 & 13.6 \\
\hline \multicolumn{3}{|c|}{ Antiepileptic drugs } \\
\hline No & 238 & 69.0 \\
\hline Yes & 107 & 31.0 \\
\hline \multicolumn{3}{|l|}{ Smoke } \\
\hline No & 342 & 99.1 \\
\hline Yes & 3 & 0.9 \\
\hline \multicolumn{3}{|l|}{ Diabetes } \\
\hline No & 341 & 98.8 \\
\hline Yes & 4 & 1.2 \\
\hline \multicolumn{3}{|l|}{$\mathrm{N}^{\circ}$ visits } \\
\hline 1 & 146 & 42.3 \\
\hline 2 & 59 & 17.1 \\
\hline 3 & 50 & 14.5 \\
\hline 4 & 36 & 10.4 \\
\hline 5 & 22 & 6.4 \\
\hline $6+$ & 32 & 9.3 \\
\hline
\end{tabular}

Table 2: BoP accounting for patients group: mean, median, and test trend

\begin{tabular}{lrrrc}
\hline & Mean & & C.I. 95\% & Median \\
\hline Patients group & & & & \\
$1(n=146)$ & 6.7 & 3.6 & 9.9 & 0.0 \\
$2(n=59)$ & 33.6 & 24.9 & 42.2 & 17.9 \\
$3(n=50)$ & 41.4 & 30.6 & 52.1 & 26.3 \\
$4(n=36)$ & 41.8 & 30.0 & 53.6 & 26.5 \\
$5(n=22)$ & 60.0 & 44.8 & 75.2 & 53.4 \\
$6+(n=32)$ & 54.0 & 41.7 & 66.4 & 44.7 \\
\hline
\end{tabular}

Trend test: $p<0.001$

\section{Discussion}

Daily oral hygiene contributes to the prevention of dental diseases but presents challenges to SCPs who are unable to perform preventive oral care themselves and rely on caregivers for asssistance, ${ }^{9}$ suggesting that they would benefit from frequent oral health assessments. ${ }^{2,7}$ 
Table 3: Mean BoP at baseline and last visit, by patients group

\begin{tabular}{clll}
\hline & Baseline $(n=199)$ & Last visit $(n=199)$ & $p$-value \\
\hline Patients group & & & \\
$2(n=59)$ & 33.6 & 5.0 & $<0.001$ \\
$3(n=50)$ & 41.4 & 4.3 & $<0.001$ \\
$4(n=36)$ & 41.8 & 0.9 & $<0.001$ \\
$5(n=22)$ & 60.0 & 1.5 & $<0.001$ \\
$6+(n=32)$ & 54.1 & 0.3 & $<0.001$ \\
Total $(199)$ & 43.2 & 2.9 & $<0.001$ \\
\hline
\end{tabular}

"Wilcoxon matched-pairs signed-rank test

With this remark, we tried to design the program with the triple aim of improved care, improved health, and lower costs per capita ${ }^{20}$ developing specific, measurable, achievable, relevant, and time-bound goals. Being a public community dentistry unit, such a study design enables us to provide to the Health District Board a recurrent report about this registry with the aim to grant human and financial resources to it.

In this study, we evaluated the effectiveness of an oral hygiene program for people with disabilities: we recruited subjects from the perspective of the aim of the study. People with baseline BoP $=0$ have been excluded from our cohort because they were not eligible for any oral hygiene intervention. In the same way, when a patient reached the recovery ( $\mathrm{BoP}=0$ after the first visit), we censored the observation time, apart from any further visit at a later time. Our study shows an improvement in SCPs' oral health status provided by a targeted intervention and represents the first report evaluating such an outcome in our region.

This portable outreach program could represent a model of a "SEAMLESS" approach to special care dentistry: ${ }^{21}$ Seamless care with a shared approach among the different elements of dental services, carers in DCDs, and all health professionals; Education and training of dental hygienists, carers, tutors, and patients focusing on the preventive approach to oral health. Improvements in oral health care can be obtained by encouraging carers to regularly examine the mouth of the person they are caring for ${ }^{22}$ In this framework, we provide oral healthcare educational meetings to all parents and caregivers with the aim to improve the oral health knowledge and skills inside family homes and institutional settings and to motivate to maintain oral health of SCPs; Equality of Access to care: such a portable outreach program minimizes future oral disease and grants a better recruitment and efficiency reducing dental anxiety and the time requested for the needed treatment; Multidisciplinary working underlining the importance of oral care to general health; Liaison enables patients, parents/tutors to work in partnership to facilitate access to tailored oral treatments; Empowerment performed by a really patient-centered oral care planning; Special care dentistry with the aim of maintaining comfort, dignity, and self-esteem and whenever possible independence and self-care; and improved Service provision and development by identifying the barriers and providing education and intervention.

In addition to delivering services meeting those in need at the locations where those in need are, outreach has an educational role including identification of underserved population and referral to services. $^{2}$

From this point of view, the limitation of this program is that the burden of the oral health issues in the target population besides dental hygiene (e.g., treatment of carious lesions, prosthetics) cannot be completely satisfied by the public dental services.

Previous studies highlighted that the oral health needs of SCPs are complex. Physical conditions required for clinical examination, demographic and clinical variables, study population size, and inclusion criteria vary greatly throughout studies reported in the literature in which investigators evaluated the oral health status and may account for variability in the reported prevalence of oral health problems. Variation in study design, the demographics of the study population, and definitions of oral health status indicators limit our ability to compare our results directly with those of other studies or of the general population. ${ }^{1,4-7}$

Next step of our study will focus on the oral health achievements over time, by monitoring the time trend of the improvements highlighted in this work.

\section{Conclusion}

Ten years later the onset of this program, the recommendations facing the burden of oral healthcare needs of SCPs spread by the Italian Ministry of Health stress that the preventive oral health services provided by the dental hygienists involving patients, carers, and parents could ultimately reduce health and oral healthcare costs. $^{23}$

Our results would represent an increased focus to incorporate dental hygiene professionals as a part of the team to provide a comprehensive health and oral healthcare plan to treat the needs of this underserved population.

\section{Clinical Significance}

\section{Scientific Rationale for the Study}

People who have greater health needs receive the least amount of care, and dental care is one of the most unmet needs. To face these inequalities, a portable outreach oral health program consisting of recurrent professional oral hygiene sessions in the daytime centers for disabled people was planned in 2009 to date.

\section{Principal Findings}

The chosen periodontal index (bleeding on probing) decreased over time and the difference was highly statistically significant.

\section{Practical Implications}

This portable outreach program provides:

- a facilitated access to oral health treatment,

- an augmented compliance of the oral hygiene sessions,

- a progressive time reduction of each session, and

- a measurable progressive improvement in the periodontal health providing a better quality of life and a reduction of a (frequently) requiring general anesthesia invasive treatment.

\section{References}

1. Stiefel DJ. Dental care considerations for disabled adults. Spec Care Dentist 2002;22(3):26S-39S.

2. Dougall A, Fiske J. Access to special care dentistry, part 1. Access. Br Dent J 2008;204(11):605-616.

3. Couto $P$, Almeida Pereira $P$, Nunes $M$, et al. Characterization of oral health in a Portuguese population with mild intellectual disability. Spec Care Dent 2019;39(3):289-305. DOI: 10.1111/scd.12376.

4. Feldberg I, Merrick J. Intellectual disabilities and dental services: experience from Israel. Frontiers Public Health 2014;2(Article 133):1-3. 
DOI: 103389/fpubh.2014.00133 [cited 2017 June 12]. Available from: https://www.ncbi.nlm.nih-gov/pubmed/25325047.

5. Anders PL, Davis EL. Oral health of patients with intellectual disabilities: a systematic review. Spec Care Dentist_2010;30(3):110-117. DOI: 10.1111/j.1754-4505.2010.00136.x.

6. Fisher K. Is there anything to smile about? A review of oral care for individuals with intellectual and developmental disabilities. Hindawi Publishing Corporation Nursing Research and Practice Volume. 2012:1-6. DOI: 10.1155/2012/860692 [cited 2017 July 26]. Available from: https://www.ncbi.nlm.nih-gov/pmc/articles/PMC3317070/.

7. Terzaghi D. Improving the oral health of seniors and persons with disabilities. United States 2015. National Association of States United for Aging and disabilities_NASUAD 2015 [cited 2017 Aug 07]. Available from: NASUAD website.

8. Chung ET, Kim JY, Chung SC. A survey of the oral health and the dental needs of handicapped patients in Korea. J Korean Acad Oral Health 1998:12:47-78.

9. Shin DK, Jeong SH, Park JH, et al. A study of oral health status and it's related factors among disabled people in Korea. J Korean Acad Dent Health 2007;31:248-262.

10. Morgan JP, Minihan P, Park A, et al. The oral health status of 4,732 adults with intellectual and developmental disabilities. J Am Dent Assoc 2012;143(8):838-846. DOI: /10.14219/jada.archive.2012.0288.

11. Kuijken NM, Naaldenberg J, Nijhuis-van der Sanden MW, et al. Healthy living according to adults with intellectual disabilities: towards tailoring health promotion initiatives. J Intellect Disabil Res. 2016;60(3):228-241. DOI: 10.1111/jir.12243.

12. Hart JT. The inverse care law. The Lancet 1971;297(7696):405-412. DOI: 10.1016/S0140-6736(71)92410-X.

13. Naseem M, Shah AH, Khiyani MF, et al. Access to oral health care services among adults with learning disabilities: a scoping review. Annali di Stomatol 2016;7(3):52-59. DOI: 10.11138/ads/2016.7.3.052.

14. Italian National Institute of Statistics ISTAT. "Inclusione sociale delle persone con limitazioni funzionali, invalidità o cronicità gravi" realised in the project "Sistema di Informazione Statistica sulla Disabilità", through an agreement (2011) between ISTAT and the Ministry of labour and social policies. 21 July 2015 [cited 2017
Dec 22]. Available from: http://search.istat.it/search?q=+\%E2\%80 $\% 9$ CInclusione+sociale+delle+persone+con+limitazioni+ funzionali\%2C+invalidit $\% \mathrm{C} 3 \% \mathrm{~A} 0+\mathrm{O}+$ cronicit $\% \mathrm{C} 3 \% \mathrm{~A} 0+$ gravi $\%$ E2\%80\%9D\&output=xml_no_dtd\&client=istat_fe\&proxystyle sheet=istat fe \&sort=date $\% 253$ AD $\% 253$ AL\%253Ad1\&oe=UTF-8 \&ie $=$ UTF.

15. Iannucci L, Gargiulo L. Access to dental care and dental health in Italy year 2013. Socio-demographic and environmental statistics directorate Istat-National Institute of Statistics 2015 [cited 2017 Apr 21].

16. Italian National Institute of Statistics-ISTAT. Global activities limitations indicator. In: Rapporto Osservasalute 2016; 234-235 [cited 2017 June 22].

17. Rosano A, Mancini F, Solipaca A. Poverty in people with disabilities: indicators from the capability approach. Social Indicators Research 2009;94(1):75-82. DOI: 10.1007/s11205-008-9337-1.

18. Association of State \& Territorial Dental Directors (ASTDD). Best practice approaches for state and community oral health programs. Oral health of children, adolescents and adults with special health care needs report. Best practice criteria 2016 [cited 2017 Dec 22]. Available from: http://www.astdd.org/best-practice-approachreports.

19. Ainamo J, Bay I. Problems and proposals for recording gingivitis and plaque. Int Dent J 1975;25(4):229-235.

20. Institute for Healthcare Improvement. Triple aim for populations 2016 [cited 2019 May 5]. Available from: www.ihi.org/Topics/TripleAim/ pages/Overview.aspx.

21. Lewis D, Fiske J, Dougall A. Access to special care dentistry, part 7. Special care dentistry services: seamless care for people in their middle years_part 1. Br Dent J 2008;205(6):305-317. DOI: 10.1038/ sj.bdj.2008.803.

22. Fickert NA, Ross D. Effectiveness of a caregiver education program on providing oral care to individuals with intellectual and developmental disabilities. Intellect Dev Disabil 2012;50(3):219-232. DOI: 10.1352/1934-9556-50.3.219.

23. Ministry of Health. Indications to take over special care persons who need oral treatment [cite 2019 May 31]. Available from: www.salute. gov.it/imgs/C_17_pubblicazioni_2842_allegato.pdf. 